\title{
Etiologies, risk factors and outcomes of dental pulp necrosis
}

\author{
Maha Ali Abdulwahab ${ }^{1 *}$, Musab Saeed Alqahtani ${ }^{2}$, Abeer Assaf Alshammari ${ }^{3}$, \\ Suzan Essam Jiffri ${ }^{4}$, Abdulrahman Mohammed Alasim ${ }^{5}$, Fahad Majed Alsharidah ${ }^{5}$, \\ Naif Ibrahim Alqunfuthi ${ }^{6}$, Batoul Abdulmalek Alnamnakani ${ }^{7}$, Raghad Saeed Alshahrani ${ }^{8}$, \\ Norah Abdullah Alshahrani ${ }^{8}$, Rian Naser Aljohani', Meshall Abdul-Lattif Almintakh ${ }^{10}$
}

\author{
${ }^{1}$ North Jeddah Specialist Dental Center, King Abdullah Medical Complex, Jeddah, Saudi Arabia \\ ${ }^{2}$ College of Dentistry, Vision Colleges, Riyadh, Saudi Arabia \\ ${ }^{3}$ Dental Department, Ministry of Health, Dammam, Saudi Arabia \\ ${ }^{4}$ General Dentist, Safaa Albarad Dental Clinic, Jeddah, Saudi Arabia \\ ${ }^{5}$ College of Dentistry, King Saud University, Riyadh, Saudi Arabia \\ ${ }^{6}$ College of Dentistry, Jazan University, Jazan, Saudi Arabia \\ ${ }^{7}$ College of Dentistry, King Abdulaziz University, Jeddah, Saudi Arabia \\ ${ }^{8}$ College of Dentistry, King Khalid University, Abha, Saudi Arabia \\ ${ }^{9}$ Dental Department, Dr Erfan \& Bagedo General Hospital, Jeddah, Saudi Arabia \\ ${ }^{10}$ Dental Department, Khulais General Hospital, Khulais, Saudi Arabia
}

Received: 14 November 2021

Accepted: 29 November 2021

\section{*Correspondence:}

Dr. Maha Ali Abdulwahab,

E-mail: mahaaabbas84@gmail.com

Copyright: ( ) the author(s), publisher and licensee Medip Academy. This is an open-access article distributed under the terms of the Creative Commons Attribution Non-Commercial License, which permits unrestricted non-commercial use, distribution, and reproduction in any medium, provided the original work is properly cited.

\begin{abstract}
Dental pulp necrosis is referred to the situation in which the teeth biologically die due to bacterial infection or without bacterial infection. Dental pulp necrosis can be due to a chronic progression of pulpitis, in which the tissue of soft pulp in the tooth dies due to several causes such as trauma or severe bacterial infection. Untreated cavities, multiple invasive treatments for the tooth, and pathological ischemia for dental pulp are among the most common causes for the necrosis process. Usually, the first symptom of dental pulp necrosis is the irritant pain in the tooth only or the surrounding area because of the inflammation. The grade of pain ranges from mild, moderate, to severe pain according to the size of the damage, followed by swelling and discomfort in chewing due to the pressure on the nerve root at the base of the tooth. We aim to review the different etiologies, risk factors, correlations, and clinical outcomes associated with pulp necrosis. To our knowledge, this article is going to be the first comprehensive review of dental pulp necrosis.
\end{abstract}

Keywords: Dental pulp necrosis, Pulpitis, Dental trauma, Dental infection

\section{INTRODUCTION}

Dental pulp necrosis is referred to the situation in which the teeth biologically die due to bacterial infection or without bacterial infection. Dental pulp necrosis can be due to a chronic progression of pulpitis, in which the tissue of soft pulp in the tooth dies due to several causes, such as trauma or severe bacterial infection. ${ }^{1}$ Untreated cavities, multiple invasive treatments for the tooth, and pathological ischemia for dental pulp are among the most common causes for the necrosis process. ${ }^{2}$ Usually, the first symptom of dental pulp necrosis is the irritant pain in the tooth only or the surrounding area because of the inflammation. The grade of pain ranges from mild, moderate, to severe pain according to the size of the damage, followed by swelling and discomfort in chewing due to the pressure on the nerve root at the base of the tooth. ${ }^{3}$ Nerve supply to the teeth is mainly from the superior alveolar nerves and inferior alveolar nerves, branches of the trigeminal nerve. ${ }^{4}$ Inflammation to the alveolar nerves can refer to trigeminal ganglion of the 
trigeminal nerve causing acute pain known as trigeminal neuralgia. ${ }^{5}$ The pathophysiology of dental pulp necrosis occurs in the following sequence. At first, the tooth gets exposed due to severe trauma penetrating the enamel and dentin, followed by bacterial penetration to the pulp and gingiva. Secondly, the healthy pulp tissue secretes inflammatory mediators against bacterial infection. The inflammatory reaction causes swelling and pain, which causes pressure on the nerve root at the tooth's base, depriving it of nutrients. The blood flow to the injured tooth is affected due causing ischemic necrosis, followed by pulp necrosis. ${ }^{6}$

The diagnosis of dental pulp necrosis requires an X-Ray to visualize the tooth. Ice-cold and hot tests are used to examine the nerve sensation. ${ }^{7}$ The treatment lines are majorly invasively followed by supportive medical treatments. The infected and damaged pulp is removed, followed by cleaning the tooth's chamber, and the space is filled with prosthetic material such as medical cement. Finally, the superficial aspect of the tooth is covered by a crown. $^{8}$ In cases that the root canal cannot be saved, extraction of the tooth will be the gold standard to prevent further disease progression. ${ }^{8} \mathrm{We}$ aim to review the different etiologies, risk factors, correlations, and clinical outcomes associated with pulp necrosis. To our knowledge, this article is going to be the first comprehensive review of dental pulp necrosis.

\section{LITERATURE REVIEW}

This literature review is based on an extensive literature search in Medline, Cochrane, and EMBASE databases which was performed on $15^{\text {th }}$ October 2021 using the medical subject headings (MeSH) or a combination of all possible related terms, according to the database. To avoid missing potential studies, a further manual search for papers was done through Google Scholar while the reference lists of the initially included papers. Papers discussing etiologies, risk factors and outcomes of dental pulp necrosis were screened for useful information. No limitations were posed on date, language, age of participants, or publication type.

\section{DISCUSSION}

\section{Microbiology and pathophysiology}

The literature correlates between the Streptococci family and lactobacillus in the process of enamel demineralization. ${ }^{9}$ Enamel demineralization is when the enamel gets gradually degenerated, exposing the dentin denying underlying it. The bacteria play an essential role in sugar formation, depending on intrinsic and extrinsic factors such as $\mathrm{pH}$ and polysaccharides genesis, necessary for fermentation and bacterial spread. ${ }^{10}$ Streptococcus mutants have many sugar-transporting systems, including the phosphoenolpyruvate-phosphotransferase system, and thrive in a highly acidic medium ( $\mathrm{pH}$ less than six). In addition to that, they can extract proteins, and that respond to acid stress. The microbiological reaction of the phosphoenolpyruvate-phosphotransferase system increases the durability of the bacterial organisms to invade through the superficial and lateral layers of the enamel, followed by penetration of dentin and targeting the dental pulp. ${ }^{10}$ The bacterial penetration to the pulp initiates an immediate inflammatory response to fight against the Streptococci and Lactobacillus migration to the infected space. The white blood cells, macrophages, lymphocytes, and neutrophils migrate toward the bacterial spot inducing the antigen-antibody reaction against the organisms. The inflammatory response releases histamine, prostaglandins, bradykinins, and endothelin to vasodilate the blood vessels nourishing the infected tooth with the body's defensive organism. This reaction allows more inflammatory mediators to react rapidly to the infected pulp and resist against it. ${ }^{11}$ However, the prolonged vasodilation of dental vessels will cause swelling and edema around the soft tissue causing direct pressure. At first, the progressive force on the nerve roots at the tooth's base will cause persistent pain with jaw movement or without movement of the jaw. If the inflammatory reaction prolongs the average time, the affection will be chronic, and the compression will affect the nerve roots and permanently damage them. The average time of inflammation is dependent on several factors regarding the severity and the general health status of the infected patient. However, the average for a healthy adult would be around five days. In cases of chronic prolongation, the nerve and blood supply to the tooth will gradually diminish, and the pulp tissue will die and be necrotic. ${ }^{12}$ Therefore, at the stage of irritant pain, a complex trajectory for immediate diagnosis and treatment is necessary to prevent progression to the necrosis phase. Secondary pathophysiological events following the dental pulp infection are related to the surrounding tissue. The bacterial organisms have the liability for spreading to the healthy teeth causing similar events to it. In addition to that, the superior alveolar nerves and inferior alveolar nerves are the main supply to the upper and lower teeth. The alveolar nerves are direct branches of the trigeminal nerve. In some severe pain or severe infection cases, the pain may refer to the trigeminal ganglion causing facial pain known as trigeminal neuralgia. Systemic spread of bacterial infection through the bloodstream is uncommon for healthy people and adults. However, people with low immunity, such as geriatrics, elderly, immunocompromised patients, patients with diabetes mellitus, post-graft patients who take immunosuppressive drugs, and patients who have artificial prosthetics as knee arthroplasty, hip arthroplasty, and intervertebral disc replacement are more liable to develop systemic bacteremia and septicemia after severe infection. ${ }^{13-15}$

\section{Chronic irreversible pulpitis}

The inflammation of the pulp is divided into two major types. Firstly, reversible pulpitis, characterized by spontaneous resolution for the inflammation, regenerates itself again, growing healthy tissue. In reversible pulpitis, 
intuitive healing occurs due to several factors, including good immunity, mild infection, and early diagnosis and management with proper treatment trajectories. While in the other hand, in cases of severe diseases as in multiple teeth exposure, malnutrition, avitaminosis (Vitamin C, vitamin A, and vitamin D deficiency), minerals deficiency (calcium and phosphorus), autoimmune diseases patients (Rheumatoid arthritis, systemic lupus erythematosus, multiple sclerosis, psoriasis, and polyneuropathy) and chronic prolongation of the infection especially in the elderly. The condition may quickly turn into a chronic event resulting in irreversible unpleasant complications. In cases of irreversible pulpitis, the tissue does not generate by itself. It ends by losing the nerve supply and blood supply for the tooth, resulting in necrotic tissue formation. Irreversible pulpitis is considered to be the most common risk factor for dental pulp necrosis. ${ }^{16}$

\section{Dental trauma}

Dental trauma occurs when the tooth is displaced from its normal position. The displacement of the tooth causes apical blood supply compression. This can occur due to luxation or avulsion directly. In addition to that, the removal might lead to inflammation for the apical periodontal ligament, which is from the secondary causes in dental trauma to cause dental pulp necrosis. ${ }^{17}$ The forms of dental trauma are numerous including, trauma to the face by punching to the front, road traffic accidents, or any other traumatic event affecting the tooth and displacing it from the normal position. Despite the cause of trauma, the results are similar. However, it differs according to the severity of the trauma, e.g., in road traffic accidents, the prognosis is worse than mild trauma to the tooth. Therefore, the management should be accurate and rapid according to the case specifically and not unified. ${ }^{17}$

\section{Wrong dental invasion}

Dental pulpal necrosis can also result from wrong dental treatment, such as iatrogenic damage caused by excessive crown fixation, which may be due to extreme heat and proximity of the pulp during dental repair or rapid orthodontic activity that causes an excessive force to be applied on the tooth, result in penetration of the enamel and dentin. ${ }^{17}$ Cases of wrong dental invasive treatments are usually due to a lack of experience of the treating dentist, and in some cases, this is considered malpractice..$^{17}$

\section{Autoimmune disorders}

Patients with autoimmune disorders have a less powerful immunity system to attack against and defend against bacterial organism migration in the body. These facilities the entry of different organisms to the body from other routes. The oral and nasal cavities have the highest possibilities of getting infected since they contain the greatest concentration of Streptococci. Therefore, it is not uncommon to see immunocompromised patients get frequent dental infections, ${ }^{18}$ similarly in patients with organ transplantation (post-graft). Conditions may be regular since the patients take immune-suppressive drugs to reduce the rates of graft rejection post-transplantation.

\section{Artificial transplants}

When the inflammatory reaction occurs against the bacterial migration, the bacteria escape from areas with high blood supply and are rich in inflammatory mediators such as large vessels. Artificial transplants such as knee transplants, hip transplants, intervertebral disc replacements, and other structures are fundamental avascular components that do not have immunological responses. Therefore, it is a suitable medium for bacterial organisms to escape at those targets. The accumulation of bacterial infection at the avascular structures can penetrate the surrounding blood vessels, causing systemic bacterial infection to spread all over the body. Dental vessels can be targeted from this bacterial spread and causing severe disease for several teeth. ${ }^{19}$

\section{Diabetes mellitus}

In the literature, diabetes mellitus was linked to different oral and dental health problems. It was observed that dental issues associated with diabetes mellitus were observed at an average of 40 years old. ${ }^{14}$ Diabetes was observed to cause progression damage for root surfaces root caries. ${ }^{14}$ In diabetic patients, the systemic blood flow to the collateral branches of vessels such as in the pulp tissue is limited, resulting in ischemia to the pulp. In addition to that, it was observed that the inflammatory mediators released during inflammatory reactions in diabetic people are different from healthy individuals. The diabetic inflammatory mediators damage the oral and dental tissue and result in rapid ischemia, which increases the chances of developing dental pulp necrosis. ${ }^{14}$

\section{Symptoms and signs}

Outcomes following dental pulp infection and progression to the necrotic phase are variant. The variance is due to several factors, including the organism, number of infected teeth (one tooth or multiple teeth), age, gender, immunological response, the health status of the patient, past medical history and association with other diseases, and history of repeated infections. Following the irritant pain, among the earliest symptoms, the patient complains of crown discoloration from the progression phase of pulpitis to necrosis. Crown discoloration occurs at the early and the middle stages before the final stage of necrosis. At first, pale colors discolor the crown, such as yellow. Later on, the discoloration process becomes darker as grey, brown, and black in the final stages. More the darker, the more the case is worse. Therefore, identifying the early discoloration of the crown is a vital sign to be noticed. Early management in the early phases will be effective in treatment lines and preventing diseases progression into hopeless stages. ${ }^{20}$ 
Dental abscesses or dental fistula are among the common manifestations associated with dental infections. The abscess formation is due to Staphylococcus infection, causing the organism to deposit fibrin in the soft tissue and forming a pyogenic spot progressing into an abscess. A similar mechanism occurs for dental fistula formation but with perforation for the tissue. However, abscess formation does not always mean pulp necrosis. Confirmatory diagnosis using $\mathrm{X}$-ray is necessary to exclude any other differential diagnosis. ${ }^{20}$ In addition to that, internal root resorption may be a sign of dental pulp necrosis. However, the radiological diagnosis for this sign is not a confirmatory diagnosis for the case.

\section{Diagnosis}

Early proper diagnosis is a necessary step to plan a trajectory for successful treatment. Ways of diagnosis are several including, grey or black tooth discoloration, negative vitality of the tooth, apical and peri-apical lesions on the tooth, and altered radiographic translucency. ${ }^{21}$ Thermal tests are standard and easy methods that are helpful in the diagnosis. These tests include direct cold or hot exposure to the tooth. Normally, if the patient has a vital tooth, a painful reaction will happen due to stimulation of the pulpar nerves (branches from the superior alveolar and inferior alveolar nerves). In cases of necrotic pulp and the nerve roots are not vital, there would be no sensation. ${ }^{7}$

The pulse oximeter test is an objective test for measuring the vital supply to the tooth. Therefore, it is more accurate than the cold and hot exposure test. The pulse oximeter measures the blood oxygen saturation levels in the tooth. Decreased supply to the tooth denotes the decreased vitality of the tooth. The literature reports that the pulse oximeter is an accurate test to diagnose the necrotic pulp and differentiation between the vital tooth and non-vital tooth. $^{22}$ Magnetic resonance imaging (MRI) techniques have been used to diagnose head and neck abnormal tissue disorders. Previous studies used MRI imaging techniques to detect the vital status of dental pulp after several pathological conditions. The results were promising. The MRI was practical to differentiate between the traumatized dental patients and healthy patients. ${ }^{23}$

\section{Management and treatment}

Root canal treatment is the gold standard treatment for the necrotic dental pulp. This includes drilling and removal of the necrotizing tissue and filling it with medical filling materials such as medical cement or any other biological prosthetics. The root filling is followed by healing and regeneration of the peri-radicular tissue. However, in some cases, the regeneration process may not be an option. Majorly, if the root has incomplete development and the apex closure is not complete. In cases in which the root canal treatment is unstable. The main treatment line will be tooth extraction, followed by medical pain relievers. ${ }^{24}$

\section{CONCLUSION}

Dental pulp necrosis is commonly a progressive phase from chronic irreversible pulpitis, which has not been diagnosed for a long time or due to additional factors, including immunological disorders as in immunocompromised patients. The immunological response causes vasodilation of the dental blood vessels, causing pressure on the dental nerve roots and damaging it irreversibly. Diagnosis of the disease before the necrotic phase occurs is an essential step to propose the proper treatment. X-ray, MRI, and dental pulse oximeter are among the accurate diagnosis methods for spotting the necrotic pulp. However, the diagnosis should be in compensation for the present and the past medical history of the patient to exclude any differential diagnosis. Treatment is mainly surgically, either root canal treatment or by extraction of the tooth. Secondary medical treatment includes pain relievers.

Funding: No funding sources Conflict of interest: None declared Ethical approval: Not required

\section{REFERENCES}

1. López-Marcos JF. Aetiology, classification and pathogenesis of pulp and periapical disease. Med Oral Patol Oral Cir Bucal. 2004;9(1):58-62.

2. Koç S, Del Fabbro M. Does the Etiology of Pulp Necrosis Affect Regenerative Endodontic Treatment Outcomes? A Systematic Review and Meta-analyses. J Evid Based Dent Pract. 2020;20(1):101400.

3. Mejàre IA, Axelsson S, Davidson T, et al. Diagnosis of the condition of the dental pulp: a systematic review. Int Endod J. 2012;45(7):597-613.

4. Leston JM. Functional anatomy of the trigeminal nerve. Neurochirurgie. 2009;55(2):99-112.

5. Tripathi M, Sadashiva N, Gupta A. Please spare my teeth! Dental procedures and trigeminal neuralgia. Surg Neurol Int. 2020;11:455.

6. Yu C, Abbott PV. An overview of the dental pulp: its functions and responses to injury. Aust Dent J. 2007;52(1):S4-16.

7. Petersson K, Söderström C, Kiani-Anaraki M, Lévy G. Evaluation of the ability of thermal and electrical tests to register pulp vitality. Endod Dent Traumatol. 1999;15(3):127-31.

8. Kahler B, Rossi-Fedele G, Chugal N, Lin LM. An Evidence-based Review of the Efficacy of Treatment Approaches for Immature Permanent Teeth with Pulp Necrosis. J Endod. 2017;43(7):1052-7.

9. Larsen T, Fiehn NE. Dental biofilm infections-an update. Apmis. 2017;125(4):376-84.

10. Johnson BT, Mayo JA, Jeansonne BG. betaHemolytic Streptococci and other beta-hemolytic organisms in apical periodontitis and severe marginal periodontitis. Endod Dent Traumatol. 1999;15(3):102-8. 
11. Feng Z, Li Q, Meng R, Yi B, Xu Q. METTL3 regulates alternative splicing of MyD88 upon the lipopolysaccharide-induced inflammatory response in human dental pulp cells. J Cell Mol Med. 2018;22(5):2558-68.

12. Dabuleanu M. Pulpitis (reversible/irreversible). J Can Dent Assoc. 2013;79:d90.

13. Aldrigui JM, Cadioli IC, Mendes FM, Antunes JL, Wanderley MT. Predictive factors for pulp necrosis in traumatized primary incisors: a longitudinal study. Int J Paediatr Dent. 2013;23(6):460-9.

14. Lima SM, Grisi DC, Kogawa EM. Diabetes mellitus and inflammatory pulpal and periapical disease: a review. Int Endod J. 2013;46(8):700-09.

15. Omi M, Hata M, Nakamura N. Transplantation of dental pulp stem cells suppressed inflammation in sciatic nerves by promoting macrophage polarization towards anti-inflammation phenotypes and ameliorated diabetic polyneuropathy. J Diabetes Investig. 2016;7(4):485-96.

16. Ricucci D, Loghin S, Siqueira JF, Jr. Correlation between clinical and histologic pulp diagnoses. J Endod. 2014;40(12):1932-9.

17. Reddy LV, Bhattacharjee R, Misch E, Sokoya M, Ducic Y. Dental Injuries and Management. Facial Plast Surg. 2019;35(6):607-13.

18. Mensch K, Nagy G, Nagy Á, Bródy A. Characteristics, diagnosis and treatment of the most common bacterial diseases of the oral cavity. Orv Hetil. 2019;160(19):739-46.
19. Kim JL, Park JH, Han SB, Cho IY, Jang KM. Allogeneic Blood Transfusion Is a Significant Risk Factor for Surgical-Site Infection Following Total Hip and Knee Arthroplasty: A Meta-Analysis. J Arthroplasty. 2017;32(1):320-5.

20. Abbott PV, Yu C. A clinical classification of the status of the pulp and the root canal system. Aust Dent J. 2007;52(1):S17-31.

21. Jacobsen I. Criteria for diagnosis of pulp necrosis in traumatized permanent incisors. Scand J Dent Res. 1980;88(4):306-12.

22. Mishra S, D SS, Bhusari C. Assessing Inflammatory Status of Pulp in Irreversible Pulpitis Cases with Pulse oximeter and Dental Hemogram. J Clin Pediatr Dent. 2019;43(5):314-9.

23. Demirturk Kocasarac H, Geha H, Gaalaas LR, Nixdorf DR. MRI for Dental Applications. Dent Clin North Am. 2018;62(3):467-80.

24. Bjørndal L, Simon S, Tomson PL, Duncan HF. Management of deep caries and the exposed pulp. Int Endod J. 2019;52(7):949-73.

Cite this article as: Abdulwahab MA, Alqahtani MS, Alshammari AA, Jiffri SE, Alasim AM, Alsharidah FM et al. Etiologies, risk factors and outcomes of dental pulp necrosis. Int J Community Med Public Health 2022;9:348-52. 